\title{
Minimal impairment in a rat model of duration discrimination following excitotoxic lesions of primary auditory and prefrontal cortices
}

\author{
Shraddha Pai ${ }^{1}{ }^{\dagger}$, Jeffrey C. Erlich ${ }^{2,3}$, Charles Kopec ${ }^{2,3}$ and Carlos D. Brody ${ }^{2,3}$ \\ 1 Watson School of Biological Sciences, Cold Spring Harbor Laboratory, Cold Spring Harbor, NY, USA \\ 2 Howard Hughes Medical Institute, Chevy Chase, MD, USA \\ ${ }^{3}$ Princeton Neuroscience Institute and Department of Molecular Biology, Princeton University, Princeton, NJ, USA
}

\section{Edited by:}

Raphael Pinaud, University of Oklahoma Health Sciences Center, USA

\section{Reviewed by:}

Shaowen Bao, University of California-Berkeley, USA

Santiago Jaramillo, Cold Spring

Harbor Laboratory, USA

*Correspondence:

Shraddha Pai, The Centre for Addiction and Mental Health, Room

R28, 250 College Street, Toronto, ON, Canada M5T 1 R8.

e-mail:shraddha_pai@camh.net

\section{${ }^{+}$Present address:}

Shraddha Pai, The Krembil Family Epigenetics Laboratory, Neuroscience Department, Centre for Addiction and Mental Health, Toronto, ON, Canada
We present a behavioral paradigm for the study of duration perception in the rat, and report the result of neurotoxic lesions that have the goal of identifying sites that mediate duration perception. Using a two-alternative forced-choice paradigm, rats were either trained to discriminate durations of pure tones (range $=[200,500] \mathrm{ms}$; boundary $=316 \mathrm{~ms}$; Weber fraction after training $=0.24 \pm 0.04$ ), or were trained to discriminate frequencies of pure tones (range $=[8,16] \mathrm{kHz}$; boundary $=11.3 \mathrm{kHz}$; Weber $=0.16 \pm 0.11$ ); the latter task is a control for non-timing-specific aspects of the former. Both groups discriminate the same class of sensory stimuli, use the same motions to indicate decisions, have identical trial structures, and are trained to psychophysical threshold; the tasks are thus matched in a number of sensorimotor and cognitive demands. We made neurotoxic lesions of candidate timing-perception areas in the cerebral cortex of both groups. Following extensive bilateral lesions of the auditory cortex, the performance of the frequency discrimination group was significantly more impaired than that of the duration discrimination group. We also found that extensive bilateral lesions of the medial prefrontal cortex resulted in little to no impairment of both groups. The behavioral framework presented here provides an audition-based approach to study the neural mechanisms of time estimation and memory for durations.

Keywords: timing, perception, audition, auditory cortex, cognition, animal behavior, excitotoxins

\section{INTRODUCTION}

Specialized timekeeping mechanisms that underlie phenomena such as circadian rhythms and sound localization are wellunderstood at the neuroanatomical, local circuit, and even molecular level (Carr, 1993; Wager-Smith and Kay, 2000). This is in sharp contrast to the relatively little we understand about the mechanisms underlying the timing of intervals in the range from hundreds of milliseconds to a few hours (Mauk and Buonomano, 2004; Buhusi and Meck, 2005). This "interval timing" ability, used by humans to plan daily activities, has also been identified in several other species (Gibbon, 1977; Boisvert and Sherry, 2006; Henderson et al., 2006). In rodents, the operant Peak Interval task (Catania, 1970; Gibbon, 1977; Roberts, 1981; Buhusi and Meck, 2005; Boisvert and Sherry, 2006; Henderson et al., 2006) is a wellestablished paradigm used to assess interval timing capacity. In this paradigm, the animal learns that a response (e.g., pressing a lever) following a specific duration after stimulus presentation, is rewarded. Such an animal develops an anticipatory increase in response rate leading up to the target duration. In probe trials where the reward is omitted, the response rate decreases after the target duration has elapsed. Pharmacological lesions in animals performing the Peak Interval task have suggested the involvement of frontostriatal loops, matching patterns observed in human neuroimaging studies (Rao et al., 2001). However, the Peak Interval paradigm involves both interval estimation and production. Here we developed a paradigm that focused purely on duration estimation, to ensure that we were studying a single cognitive function. We also attempted to simplify the interpretations of our experiments by controlling for unrelated sensorimotor and cognitive variables (e.g., motivation). To this end, we developed two forcedchoice tasks involving the bisection of auditory cues: one where the relevant parameter to be bisected was tone duration and the other, tone frequency; temporal bisection has been previously used to characterize interval timing at the behavioral level (Church and Deluty, 1977).

Based on the literature from interval timing in humans, we hypothesized that timing in our task occurred via a corticocortical pathway (Rao et al., 2001; Nenadic et al., 2003; also see Figure 14). We therefore performed extensive bilateral lesions of the auditory cortex (ACx), the cortical location which neuroanatomically receives the earliest impulses from the auditory thalamus. We also performed extensive bilateral lesions of the medial prefrontal cortex (mPFC), which is required for some forms of short-term memory in the rat (Ragozzino et al., 2002; Buhusi and Meck, 2005). All lesions were conducted in both the timing and frequency groups of animals. Here we describe our behavioral framework and the results of these lesions. 


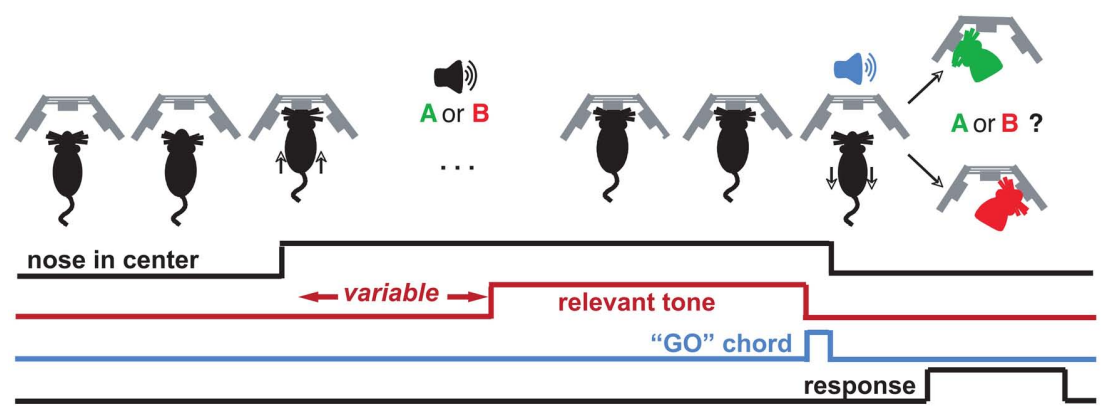

FIGURE 1 |Trial structure for the duration and frequency discrimination tasks. The behavior box contains three nose cones with which the rat interacts to advance a trial. The rat initiates the trial with a center poke, upon which he is presented with a variable-length silent period, a cue ("A or B"), and a "GO" chord, which is a permissive signal for indicating sound categorization. Decisions are indicated by a subsequent side poke (red/green silhouette). Each class of stimuli is associated with rewards on only one side (e.g., left for "short," right for "long"). Correct responses are rewarded with $24 \mu \mathrm{L}$ water, and incorrect ones are punished by omission of reward and presentation of pulsing white noise (see Materials and Methods).

\section{MATERIALS AND METHODS}

These experiments used only adult male Long-Evans rats. Animals were housed in a reverse dark-light cycle and were given free access to food; access to water was limited to $30 \mathrm{~min}$ a day. Rats were water deprived under a protocol approved by the Cold Spring Harbor Laboratory and by Princeton University Institutional Animal Care and Use Committees.

\section{HARDWARE/SOFTWARE CONFIGURATION}

All training was conducted in a training box (custom-made by Island Motion, Tappan, NY, USA) with a grill floor, enclosed in a soundproof chamber (Industrial Acoustics Company, Bronx, NY, USA). One wall of the training box contained an array of three side-by-side plastic "nose ports" or "nose pokes" (custommade by Island Motion, Tappan, NY, USA after a design by Z. F. Mainen and A. M. Zador), each shaped such that rats could insert their nose into a conical aperture facing the interior of the box (see schematic in Figure 1). Nose pokes into/out of the ports were detected by infrared beam breaks. Water was delivered via metal tubes fitted to the backs of the leftmost and rightmost ports; these tubes in turn were connected to a computer-controlled water delivery system. Beam breaks were digitally recorded via a data acquisition card (National Instruments, Austin, TX, USA) on a computer running the Real-time Linux operating system and custom, open-source software ${ }^{1}$ that detected and responded to behavioral events with a clock precision of $166 \mu \mathrm{s}$. The Real-time Linux computer was in turn controlled by a computer running custom open-source software written in $\mathrm{Matlab}^{2}$, in which the stimulus/response characteristics of each trial were configured. The walls flanking the ports were perforated and had computer-controlled speakers (Harman Kardon, Stamford, CT, USA) placed behind them. After experience training many animals, a shaping algorithm was developed that allowed adaptive training of each animal in an automated fashion (discussed in detail in the following section).

\footnotetext{
${ }^{1}$ http://code.google.com/p/rt-fsm

${ }^{2} \mathrm{http}: / /$ brodylab.princeton.edu/bcontrol
}

\section{BEHAVIOR}

Rats were trained to discriminate either pure tone durations or pure tone frequencies. In both cases, the trial structure was the same (Figure 1). An animal initiated a trial by poking its nose into the center nose port. Animals were trained to then continuously maintain their noses in the center port until a brief chord was played ("GO" signal; chord composed of 16 pure tones with base frequency $1 \mathrm{kHz}$, lasting $100 \mathrm{~ms}$ ). Nose withdrawals before the GO signal resulted in aborting of the trial, signaled by a "penalty sound" consisting of four pulses of white noise (pulse duration $=150 \mathrm{~ms}$, inter-pulse interval $=50 \mathrm{~ms}$ ) followed by $2-$ $6 \mathrm{~s}$ of white noise. After the white noise sound, aborted trials were restarted afresh. The white noise duration increased or decreased based on respective increase/decrease in recent frequency of penalty occurrence.

The initiating center poke triggered the start of a variable-delay (150 ms-1 s) silent period, followed by a single pure tone. In trials that were not restarted due to early withdrawals, the pure tone was followed by the "GO" signal, which indicated to the rat that it was now free to take its nose out of the center port and seek a 24$\mu \mathrm{L}$ water reward by nose poking into either the left or right port. The identity of the pure tone played while the animal had its nose in the center port indicated which of the two side ports held the reward. Correct responses triggered delivery of the water reward at the corresponding side port. Incorrect responses triggered a penalty sound (same parameters as penalty sound for premature nose withdrawals). For rats trained in duration discrimination, the pure tone had a frequency of $11.3 \mathrm{kHz}$ in all trials, but had a different duration in different trials. On each trial, the logarithm of the pure tone duration was chosen from a uniform distribution ranging from $\log (200 \mathrm{~ms})$ to $\log (500 \mathrm{~ms})$. Durations longer than $316 \mathrm{~ms}$ (the geometric mean of 200 and 500) indicated reward in the right port, while durations shorter than $316 \mathrm{~ms}$ indicated reward in the left port. For rats trained in frequency discrimination, the pure tone always had a duration of $316 \mathrm{~ms}$, but had a frequency that varied across trials. The logarithm of the frequency was chosen from a uniform distribution ranging from $\log (8 \mathrm{kHz})$ to $\log (16 \mathrm{kHz})$. Frequencies higher than $11.3 \mathrm{kHz}$ (the geometric mean of 8 and 16) indicated reward in the right port while 
frequencies lower than $11.3 \mathrm{kHz}$ indicated reward in the left port. We refer to $11.3 \mathrm{kHz}$ and $316 \mathrm{~ms}$ as the "midpoint" or "categorization boundary" in each task. Training: each rat was first trained to categorize single pairs of stimuli at the endpoints of the stimulus range (200 and $500 \mathrm{~ms}$ in DURATION; 8 and $16 \mathrm{kHz}$ in FREQUENCY). To proceed to the next stage in training, the following criteria were required to be met: performance accuracy $>87 \%$ for last 30 trials, with the average number of abortions per completed trial $<1.3$, for preceding 25 trials. The rate of aborted trials varied considerably among animals; depending on the animal, 10-60+\% of the trials could be aborted.

When performance criteria were achieved, the single stimulus pair was changed to a pair closer to the midpoint, with the geometric mean of the pair being used in any session always remaining at the task's midpoint. We termed this the "sharpening" phase of training. Based on achieving the performance criterion at each step, stimulus pairs were successively moved closer and closer to the midpoint until the stimulus pair was 252 and $396 \mathrm{~ms}$ in the timing task, and 10.2 and $12.6 \mathrm{kHz}$ in the frequency task. These limits were chosen because most rats could reach the corresponding stimulus pair, but few could go beyond it. When the performance criterion was achieved at this final stimulus separation, the rat was moved into what we termed the "psychometric," or final, data collection stage of the task. In the psychometric stage, we did not use only one stimulus pair in each session, but instead on each trial chose the stimulus parameter randomly from within the task's full range, as described above.

Animals with excessive side bias (bias $>25 \%$ to any one side) or which took longer than 30 days to achieve criterion at any point in the sharpening phase of training were removed from the experiments.

\section{Data analysis}

Stimulus discriminability was quantified using the Weber ratio, using a definition from (Meck, 1986).

Weber $=\frac{s_{75}-s_{25}}{s_{50}}$

where $s_{N}$ is obtained from the fit as the stimulus that produces a leftwards response $N \%$ of the time. The numerator, $s_{75}-s_{25}$, is the just-noticeable-difference (JND), the smallest change in stimulus required to produce a substantial change in behavior (here, a $50 \%$ change in side choice). The Weber ratio is therefore the mean-normalized JND.

To more precisely compute slope from psychometric data and measure post-lesion impairment, two different functions were fitted to the behavioral data. First, Matlab's nlinfit method was used to fit a four-parameter sigmoid (eqn. 2),

$y=y_{0}+\frac{a}{1+\mathrm{e}(-(x-x 0) / b)}$

where $y$ represents the fraction of trials in which the animal responded to the left port ("short" for timing, and "low" for frequency) and $x$ represents the logarithm of the stimulus parameter (i.e., pure tone frequency or pure tone duration, depending on the task). In these fits, $y_{0}$ controls the lower bound of the curve, $\left(a+y_{0}\right)$ controls the upper bound, $x_{0}$ controls the point of inflection of the sigmoid, and $b$ controls the inverse of the slope at the point of inflection. The same data were also fitted to a line equation (eqn. 3):

$y=m x+b$

where $m$ is the slope and $b$ is the intercept at the $y$-axis. The $Q$-value (Press et al., 1992, equations 15.2.12 and 6.2.3.) was computed for each of the two fits as a measure of goodness-of-fit, and the fit with the higher $Q$-value was chosen. The linear fit was also chosen outright in the case where an animal had extremely biased responses (defined as chance performance or worse on endpoints of the psychometric range). The slope parameter of the appropriate fit was then taken to indicate the animal's behavioral sensitivity to changes in the stimulus parameter. For data combined over sessions, responses from individual sessions were first pooled and the fit was computed on this pooled response data.

\section{THREE REPLICATIONS}

Our experiments were conducted in three sets, with the following number of animals in each set: Duration $=[7,6,4]$, Frequency $=[7,7,0]$. The first set of experiments was conducted at Cold Spring Harbor Laboratory, while the second and third were conducted at Princeton University, 6 months apart. There were some differences in the task design among the three sets. In the first set of experiments, every training session began with "sharpening" trials, a phase continuing until either performance criteria for psychometric trials were met (see above) or 100 trials elapsed, whichever came first. Thereafter, psychometric trials were presented till the end of the session. This switching from sharpening to psychometric trials occurred even after rats reached the psychometric training stage. In contrast, once rats in sets 2 and 3 reached the psychometric stage, individual training sessions started directly with psychometric trials (i.e., without sharpening). Another difference between the sets was that criteria for inclusion of trained animals in lesion experiments were less strict in set 1 compared to those for sets 2 and 3 . In set 1 , any animal that maintained a psychometric curve for at least 2 weeks with a Weber ratio $<0.38$ it was included. In sets 2 and 3, only those rats that had $<=10 \%$ error rate at each endpoint of the stimulus range were included in the lesion experiments. Finally, lesion coordinates and infusion volumes were larger for sets 2 and 3 than for set 1 (see below). All sets led to similar outcomes, therefore we report the collective results here.

\section{EXPERIMENT 1: AUDITORY CORTEX}

Animals were trained to discriminate either duration (DURATION or TIMING group) or frequency (FREQUENCY group), as described above.

Thirteen DURATION animals were injected with the excitotoxin ibotenic acid, and four with saline. Ten FREQUENCY animals were injected with ibotenic acid and four with saline.

\section{Surgery}

The timeline of a lesion experiment is graphically depicted in Figure 2A. A rat to be lesioned was given free access to water 


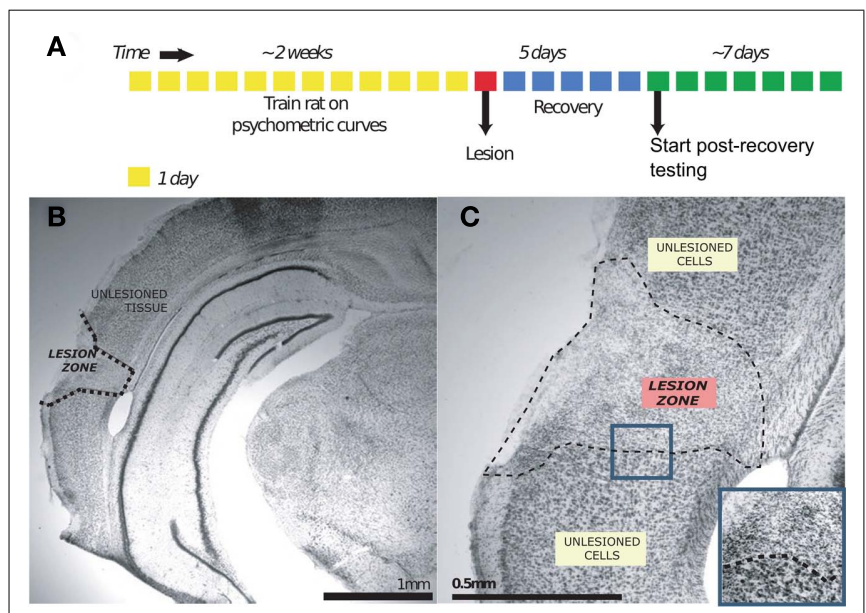

FIGURE 2 | Ibotenic acid lesion experiment. (A) Shows the timeline of the lesion experiment for an individual animal. (B) Shows the effect of ibotenic acid injection on a sample coronal section of auditory cortex, magnified in (C) [Nissl stain]. The zone of affected tissue is characterized by a disruption of the laminar cytoarchitecture of cortical tissue, and a change in the morphology of present cell nuclei from being large to being small and more disorganized [blue inset in (C)]. The boundary of this histological change is visually salient and is taken to mark the lesion extent.

the night before the lesion surgery. Just before surgery, the rat was anesthetized using isoflurane mixed in oxygen; this anesthesia was induced using $4 \%$ isoflurane and was maintained using $2 \%$ throughout surgery. At this time, ketofen $(1.3 \mathrm{mg} / \mathrm{kg})$ was also subcutaneously administered as an analgesic. Upon induction, the animal was placed into a stereotax (Kopf Instruments, Tujunga, CA, USA) atop a heating pad to maintain body temperature. Using a scalpel, an incision was made down the mid-line of the scalp and the skin flaps were clipped back. To avoid damage to the temporal muscle, the ACx was targeted from the dorsal aspect of the skull rather than through the temporal bone. This strategy required a descent angled at $30^{\circ}$ mid-line from the vertical in the coronal plane; damage to intervening tissue was avoided by use of an extremely thin $(\mathrm{OD}=10-30 \mu \mathrm{m})$ glass pipette. Target coordinates were revised part-way through the study to improve regional coverage. AP and ML coordinates (in $\mathrm{mm}$ ) are expressed relative to bregma, while the penetration depth is that (in $\mathrm{mm}$ ) of the angled-needle from dural surface. The first set (Toxin: $n=5$ duration, $n=5$ frequency; Saline: $n=2$ duration, $n=2$ frequency) involved four injection sites per hemisphere: $-3.1 \mathrm{AP}, \pm 3.53 \mathrm{ML}$, 7.06 D; -4.0 AP, 土4.66 ML, 5.08 D; -5 AP, $\pm 4.72 \mathrm{ML}, 4.96 \mathrm{D}$; $-5.5 \mathrm{AP}, \pm 4.72 \mathrm{ML}, 4.46 \mathrm{D} . \sim 81 \mathrm{~nL}$ of ibotenic acid $(10 \mathrm{mg} / \mathrm{mL}$; Tocris Bioscience, Missouri) was injected at each site. The second and third set (Toxin: $n=5$ duration, $n=5$ frequency; Saline: $n=2$ duration, $n=2$ frequency) had five injections per hemisphere: S1: $-2.50 \mathrm{AP}, \pm 3.50 \mathrm{ML}, 6.30 \mathrm{D} ; \mathrm{S} 2:-3.50 \mathrm{AP}, \pm 4.65 \mathrm{ML}, 4.40 \mathrm{D}$; S3: $-4.50 \mathrm{AP}, \pm 5.20 \mathrm{ML}, 3.50 \mathrm{D}, \mathrm{S} 4:-5.00 \mathrm{AP}, \pm 4.2 \mathrm{ML}, 5.50$ $\mathrm{D}$, and $\mathrm{S} 5:-6.00 \mathrm{AP}, \pm 4.75 \mathrm{ML}, 4.30 \mathrm{D}$. In the second set, the amount of ibotenic acid injected per site varied: S1: $138 \mathrm{~nL}, \mathrm{~S} 2$ : $110 \mathrm{~nL}, \mathrm{~S} 3: 110 \mathrm{~nL}, \mathrm{~S} 4: 110 \mathrm{~nL}$, and S5: $138 \mathrm{~nL}$. All craniotomies were made prior to any injections, and were covered with gelfoam when not in use for an injection. Injections were made using an nanoliter injector (Drummond Scientific Instruments, Broomall, PA, USA), using a custom glass pipette (shaft length $=1 \mathrm{~cm}$, tapering to $\mathrm{OD}=10-30 \mu \mathrm{m})$. Injections of ibotenic acid $(10 \mathrm{mg} / \mathrm{mL})$ or $0.9 \%$ saline were made in boluses of $\sim 28 \mathrm{~nL}$. An inter-pulse interval of $5 \mathrm{~s}$ was used, and $1 \mathrm{~min}$ was allowed to elapse between the last pulse and the raising of the needle. Finally, craniotomies were covered using a silicone elastomer (Kwik-cast; World Precision Instruments, Sarasota, FL, USA), and the skin over the scalp was sutured into place. The animal was allowed to recover in its home cage with free access to food and water for 5 days; water deprivation was recommenced on the fifth night of recovery, and behavioral testing started the following day.

\section{Post-processing and lesion scoring}

After post-lesion testing was complete, an animal was deeply anesthetized with ketamine, and then was transcardially perfused using saline, followed by $4 \%$ paraformaldehyde. The brain was removed, postfixed overnight, and coronal sections of $50 \mu \mathrm{m}$ were made using a fixed-tissue vibratome. These slices were then mounted on gelatin-subbed slides and Nissl-stained to reveal gliotic damage. Briefly, slices were selected at 100-150 $\mu \mathrm{m}$ intervals from $-2 \mathrm{AP}$ to $-7 \mathrm{AP}$ ( $\mathrm{mm}$ relative to Bregma), to match the $120 \mu \mathrm{m}$ intervals shown in the brain atlas. Light microscope images were captured at 10, 20, and 40× magnification. Gliotic regions (see Figure 2B for an example) were visually demarcated and scored onto digital copies of the rat brain atlas (Paxinos and Watson, 2007) in Adobe Acrobat Reader. Coordinates for regions of interest (ROI; here, $\mathrm{ACx}$ is defined as areas $\mathrm{A} 1, \mathrm{AuD}, \mathrm{AuV}$, and TeA) were obtained from digital images of the rat brain atlas, and the overlap between each ROI and lesions was quantified using custom-developed software in Matlab (Figure 3).

\section{Calculation of impairment}

To compute impairment, data from the last seven training sessions before the lesion surgery (BEFORE) was compared to that from the first three training sessions after the surgery and recovery (AFTER). We chose the first three post-lesion sessions as a tradeoff between maximizing the number of behavior trials obtained for data collection, while minimizing recovery effects on a timescale of days or longer. We originally intended to use the Weber ratio to quantify impairment but observed that, following lesions, the particular effect of lesions on the psychometric curve varied among animals. In particular, impairment was a combination of an effect on the slope as well as on the bias (endpoints) of the psychometric curve (see Figure 7), but the change in each varied across animals. Moreover, the Weber ratio relies on sufficient datapoints at various quartiles of response side (e.g., "25\% of stimulus X reported as 'short"'), a criterion we were unable to meet given the severe post-lesion impairment of several animals in the frequency group (e.g., post-lesion severe side bias). Conventional measures (e.g., overall accuracy) were inadequate because they suggested modest impairment when the psychometric curve suggested otherwise. We therefore decided to measure impairment as the sum of the change in both these parameters after the lesion. The IMPAIR measure was used because it was a composite measure of general performance (accuracy at end points) as well as of difficult discriminations (slope of psychometric curve). We have therefore 


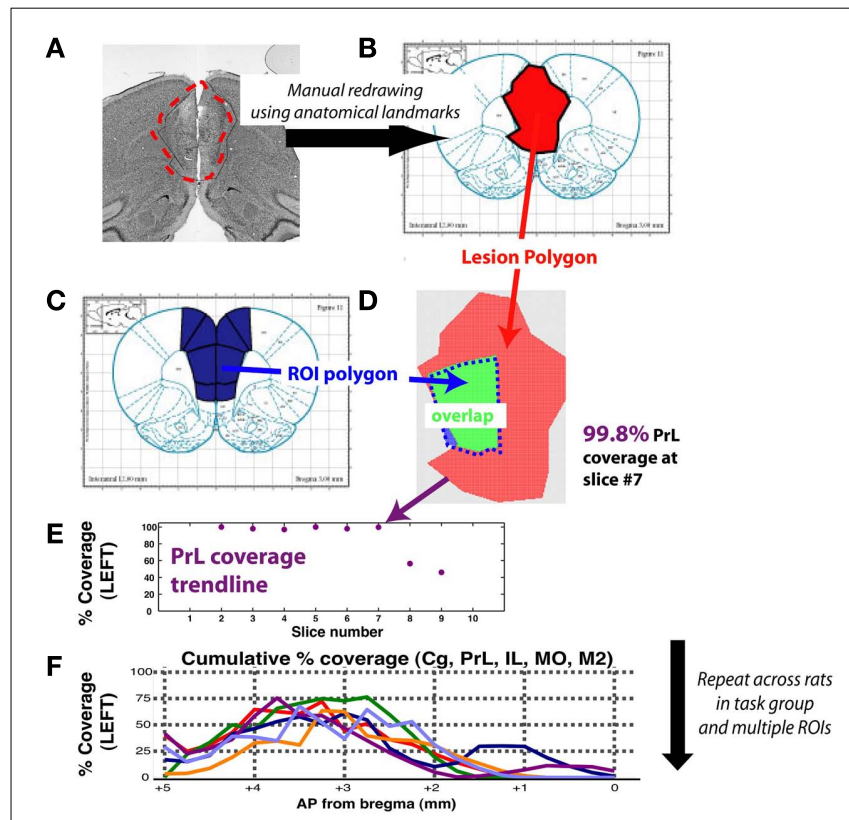

FIGURE 3 | Schematic for method of quantifying lesion extent for a single animal, and for a task group. The example shown is for prelimbic cortex, a region of medial prefrontal cortex. (A) Lesion extent was manually marked at $120 \mu \mathrm{m}$ intervals, based on gliosis evident using the Nissl stain. (B) Resulting lesion polygons were manually marked on corresponding slices of the digital rat brain atlas (Adobe Acrobat, Adobe Systems Incorporated, San Jose, CA, USA). (C) A reference set of polygons for each $\mathrm{ROI}$ was created by manually marking region extents as defined by the rat brain atlas. (D) Vertex coordinates for both lesion and ROI polygons were imported into Matlab and pixel counting was used to compute degree of overlap for each slice with each ROI. (E) Profile of slice-wise coverage of a $\mathrm{ROI}$ for a single animal. (F) Coverage trends of various prefrontal regions for each rat (trendline) of timing group. Coverage extent for missing slices was interpolated using a shape-averaging algorithm between nearest two slices. Abbreviations: $\mathrm{Cg}$, cingulate cortex; IL, infralimbic cortex; $\mathrm{MO}$, medial orbitofrontal cortex; M2, motor cortex; PrL, prelimbic cortex.

selected simple elements derived from the parameters used to fit our psychometric data - slope and accuracy change - and have used a summation of them to create a function that best measures impairment in this dataset. The IMPAIR measure agrees well with the degree of impairment visually apparent in psychometric data before and after lesions (Figures 6,7,10, and 11).

Algorithm. For each epoch (i.e., BEFORE or AFTER), data were fit to a four-parameter sigmoid (eqn. 2) as well as to a twoparameter line (eqn. 3), and the lowest-parameter fit consistent with the data was chosen (see above).

IMPAIR was defined as:

$$
\begin{aligned}
\text { IMPAIR } & =\Delta \mathrm{SLOPE}+\Delta \text { HITRATE } \\
\Delta \mathrm{SLOPE} & =\operatorname{SLOPE}_{\mathrm{BEFORE}}-\mathrm{SLOPE}_{\mathrm{AFTER}} \\
\mathrm{SLOPE} & = \begin{cases}\max (\text { slope } \forall x \in \text { stimulusrange }) & \text { if sigmoid fit } \\
m & \text { if linear fit }\end{cases}
\end{aligned}
$$

when the sigmoid fit to the data had its inflection point within the stimulus range used, the above expression for sigmoids simplifies to $a / 2 b$. For a few data sets, however, the fitted inflection point fell outside the stimulus range, in which case the maximum slope within the stimulus range was used.

$$
\Delta \text { HITRATE }=\text { ENDPOINTS }_{\text {BEFORE }}-\text { ENDPOINTS }_{\text {AFTER }}
$$

Where ENDPOINTS are the fraction of trials correct at stimulus range endpoints; operationally these were defined thus:

\section{ENDPOINTS \\ $= \begin{cases}\text { fraction } \operatorname{correct}(x \leqslant 9.9 \mathrm{kHz} \mid x \geqslant 14 \mathrm{kHz}) & \text { if DURATION } \\ \text { fraction } \operatorname{correct}(x \leqslant 264 \mathrm{~ms} \mid x \geqslant 425 \mathrm{~ms}) & \text { if FREQUENCY }\end{cases}$}

\section{EXPERIMENT 2: MEDIAL PREFRONTAL CORTEX}

Unless otherwise specified, procedures for this experiment are as described in the previous experiment.

Six DURATION animals were injected with the excitotoxin ibotenic acid. Five FREQUENCY animals were injected with toxin. No saline injections were performed.

\section{Surgery}

Craniotomies were made at $+3.0 \mathrm{AP}, \pm 0.50 \mathrm{ML}$ (all units are in $\mathrm{mm}$ and coordinates are relative to bregma). Ibotenic acid was injected bilaterally into the mPFC (one site per hemisphere): +3.0 $\mathrm{AP}, \pm 0.50 \mathrm{ML},-4.10 \mathrm{DV}$. Approximately $304 \mathrm{~nL}$ of ibotenic acid was injected at each site. For scoring, $\mathrm{mPFC}$ was defined as areas PrL and IL in the rat brain atlas $(5.64-2.52 \mathrm{~mm}$ AP relative to Bregma; Paxinos and Watson, 2007).

Post-processing and lesion scoring conducted as in auditory cortex experiments.

\section{RESULTS}

\section{PRE-LESION BEHAVIOR}

Rats in the DURATION group successfully learned to discriminate tone durations up to psychophysical threshold (Figure 4). This ability is quantified using the Weber ratio, a standard psychophysical quantity measuring uncertainty in perceptual discriminability (see Materials and Methods). Figure 4B quantifies the variability in session- and individual-specific Weber ratios from a group of animals prior to lesion; the average Weber ratio was $0.24(\mathrm{SD}=0.04)$, translating to a just-noticeable-difference (JND) of $\approx 75 \mathrm{~ms}$. Individuals vary from 0.18 (JND is $\approx 58 \mathrm{~ms}$ ) to 0.32 (JND is $\approx 102 \mathrm{~ms}$; Figure 4C). Setting the intensity of the pure tone cue to zero leads to a drop to chance performance, confirming that this task is auditory in nature (Figure 4D; $20 \%$ of the trials were randomly selected to be probe trials), and that other behavioral timing markers were not being used.

Similarly, the frequency group learned to discriminate frequencies up to psychophysical threshold (Figure 5); for this group, stimuli were also spaced uniformly in log-space. The average Weber ratio of trained animals in this group is around 0.16 , translating to a JND of $1.8 \mathrm{kHz}$. Across different animals, the Weber ratio ranged from 0.11 (JND $\approx 1.3 \mathrm{kHz}$ ) to 0.33 (JND $\approx 3.7 \mathrm{kHz}$ ). Similar to the duration task, setting the intensity of the cue to zero results in performance at chance levels (data not shown). 


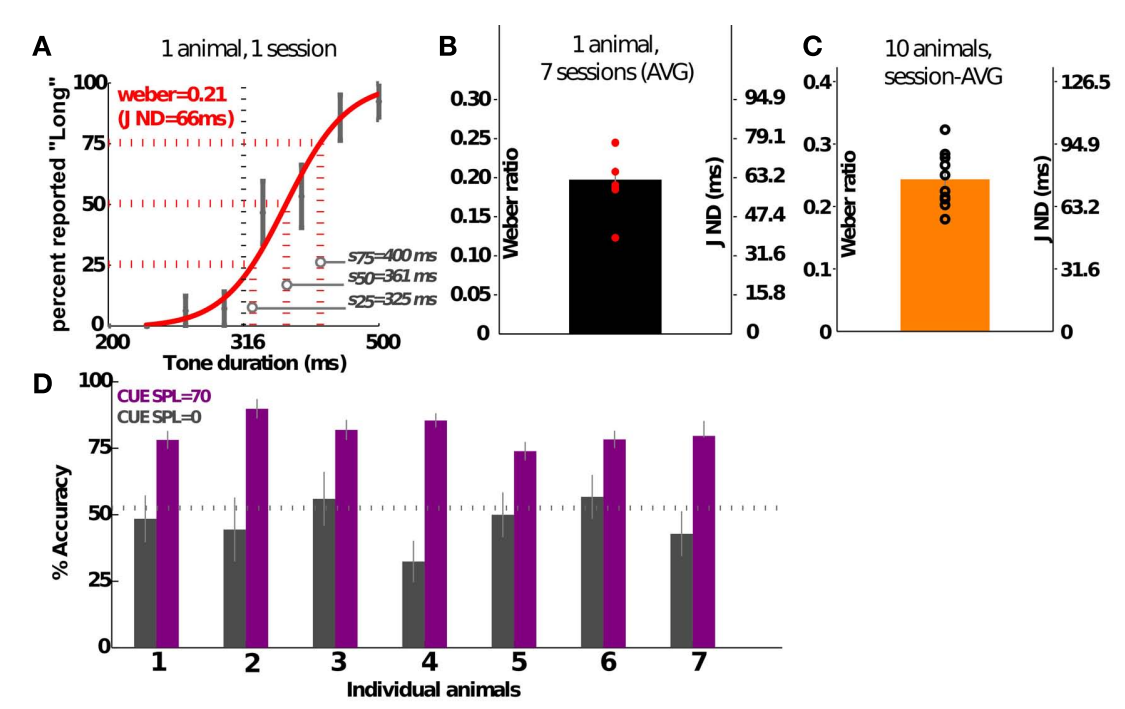

FIGURE 4 | Psychophysical characterization of the group performing duration discrimination. (A) Shows the psychometric curve generated from a single training session of a representative individual. Error bars show SEM. (B) Shows the variability in Weber ratio across 1 week's training of the animal shown in (A). (C) Shows the group average of a sample of 10 animals. (D) Shows the drop in performance to chance levels when the cue intensity is dropped to 0 in probe trials $(20 \%$ of the trials, randomly selected); data shown for seven individuals over three sessions. Three of the animals were performing in psychometric sessions, and the rest were discriminating individual tone pairs. Values shown are mean (SEM). JND, just-noticeable-difference (see Materials and Methods).
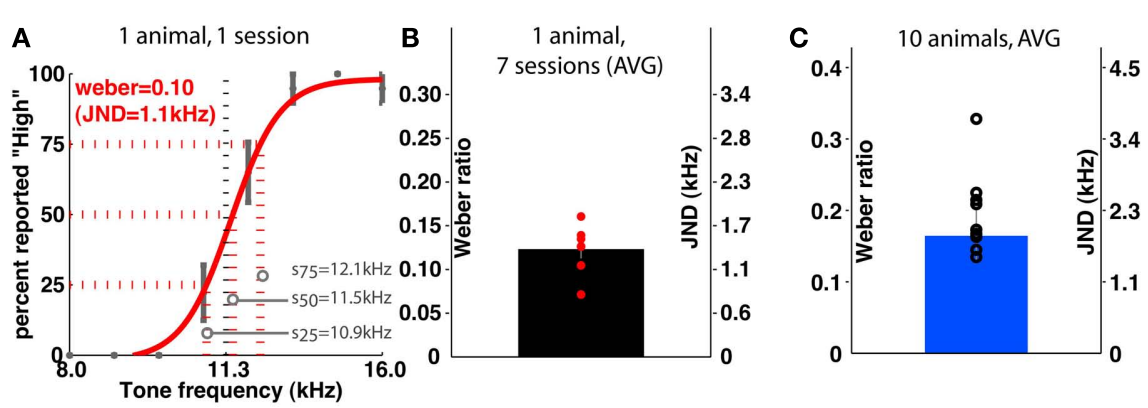

FIGURE 5 | Psychophysical characterization of the group performing frequency discrimination. (A) Shows the psychometric curve generated from a single training session of a representative individual. (B) Shows the variability in Weber ratio across 1 week's training of the animal shown in (A). (C) Shows the group average of a sample of 10 animals. JND is just-noticeable-difference (see Materials and Methods).

\section{EXPERIMENT 1: AUDITORY CORTEX LESIONS}

Among those treated with ibotenic acid, there were 9 animals in the DURATION group and 10 in the FREQUENCY group. Additionally, four animals from each group were injected with saline. Figure 6 shows representative post-lesion changes in psychometric curves for the two groups, along with IMPAIR scores. An individual's IMPAIR value is the sum of the post-lesion change in the slope of the psychometric curve and of the change in overall accuracy at the endpoints of the stimulus range (see Materials and Methods for details and justification). This metric combines the degree of impairment in finer discriminability (slope) as well as in accuracy at stimulus endpoints, and was chosen because individual animals varied in the extent to which each was affected. Figure 7 shows IMPAIR measures alongside psychometric data for all ibotenic acid-lesioned animals in this experiment. We observed tremendous variability in the degree of impairment, even in animals within a task group, and hypothesized that this variability in impairment may in turn reflect the individual variability in damage to the ACx. We therefore quantified the bilateral tissue damage to the $\mathrm{ACx}$ as well as to surrounding brain areas (Figure 8; see Materials and Methods section for algorithm). Figure 9 shows the degree of impairment plotted against bilateral lesion extent in the ACx. In general, animals of both groups were similarly covered, although two animals in the FREQUENCY group were more greatly covered than all DURATION animals. It is visually apparent that in both groups, as coverage of ACx increased, so did the degree of impairment; however, per unit coverage, there is greater impairment in the frequency group than in the timing group. This effect has been quantified by a linear regression on percent coverage of 


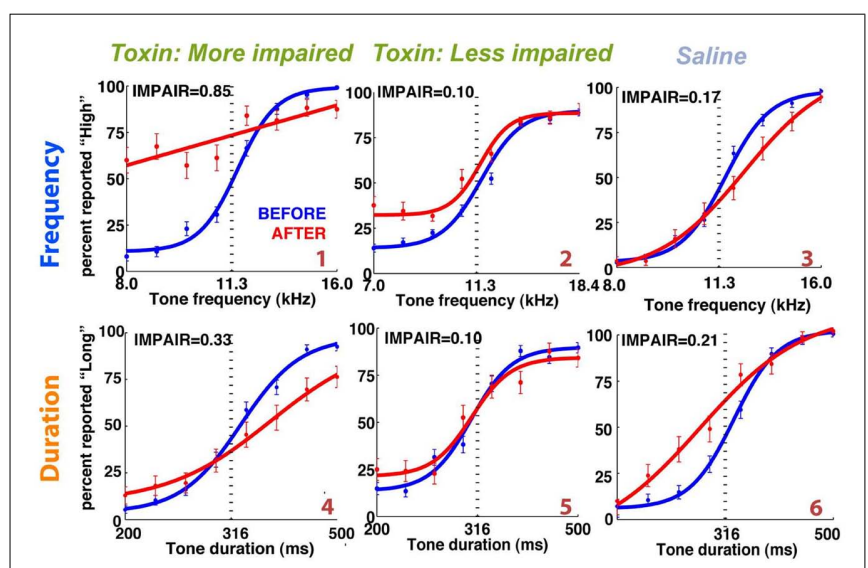

FIGURE 6 | Representative individual psychometric curves of frequency (top) and duration (bottom) group animals. Each panel shows the performance of an animal before (blue) and after (red) injections bilaterally into the auditory cortex, with the degree of impairment (IMPAIR) shown in the top-left corner. The coverage for each animal (identified by the red numeric in the bottom-right) is shown in Figure 9). Pairs of [stimulus duration/frequency, side choice] were fitted using a logistic function, or a line, to generate psychometric curves. Individual points along a curve are averages of binned values \pm SEM in log(stimulus) space. See Section "Materials and Methods" for details and justification of IMPAIR score.

the ROI $(c v g)$ as well as on an interaction term of coverage and task type:

$\operatorname{IMPAIR}=\alpha(\mathrm{cvg})+\beta($ tasktype $* \mathrm{cvg})+\gamma$

with tasktype coded as frequency $=-0.5$ and duration $=+0.5$. The effect of coverage was significantly greater than zero $(\alpha=0.58$; $95 \% \mathrm{CI}=[0.14,1.02])$ as was the interaction between coverage and the type of task $(\beta=0.52 ; 95 \% \mathrm{CI}=[0.18,0.85])$.

Animals injected with saline showed variable impairment (Figure 9, diamonds) despite lacking any visible histopathology of brain tissue (data not shown); this impairment may be due to the lapse in training during recovery from surgery, to non-specific brain injury caused by $\sim 1.0 \mu \mathrm{L}$ saline infusion, or to some other aspect of surgery.

\section{EXPERIMENT 2: MEDIAL PREFRONTAL CORTEX LESIONS}

The training timeline and general surgical/histological procedures for this experiment are identical to those used for the ACx lesions; the only difference is that for this experiment, toxin and saline injections were targeted to cover the mPFC (areas PrL and IL). Figure 10 shows the psychometric curves for representative animals in each group following the lesion, with the corresponding impairment score (IMPAIR) for each; see Figure 11 for all individual IMPAIR scores and psychometric curves. Lesion extent was quantified for both groups (Figure 12). Figure 13 shows impairment as a function of tissue coverage. Both groups showed little to no post-lesion impairment despite extensive bilateral coverage of mPFC. A linear regression showed neither a significant effect of coverage itself $(\mathrm{cvg}=0.17 ; 95 \% \mathrm{CI}=[-1.61,1.95])$, nor that of an interaction of task and coverage (tasktype ${ }^{*} \operatorname{cvg}$ coefficient $=0.06$; $95 \% \mathrm{CI}=[-0.27,0.38])$.

\section{DISCUSSION}

We developed two auditory perceptual decision-making tasks in rats, one where rats discriminate tone durations and the other, tone frequency. Using these tasks, we have presented lesion-based evidence for neuroanatomical dissociation in the neural pathways mediating the two behaviors. In both tasks, performance is based on auditory cues - inescapably so for the frequency discrimination task, and confirmed for the duration task (Figure 4D). The auditory nature of the two tasks constrains the relevant neural structures underlying the behaviors to those stemming from auditory pathways. We performed parallel lesions in separate groups of rats, each group trained in one of the two tasks. The two tasks were matched in several sensorimotor as well as cognitive aspects (e.g., maintaining a nose-in-center position, randomized stimuli during psychometric phase of task). This balanced design helps to dissociate experimental effects on temporal processing per se from indirect influences such as changes in arousal, motivation, attention, or other cognitive variables. Such confounds have challenged other studies of temporal processing (Drew et al., 2007; Ward et al., 2009). While we have used the frequency discrimination task as a control for the timing task here, the former is also a behavioral framework in its own right, for the neurobiological study of the basis of tone frequency perception and memory. We found that following lesions, animals varied in the degree to which impairment affected simple or difficult discriminations (for example, see Figure 7); the former type of impairment is readily measured by endpoints while the latter is more sensitively measured by the slope. Moreover, we found that overall rate of accuracy did not reflect the individual degree of impairment visible in the pre- and post-lesion psychometric curves. We therefore developed a composite measure (IMPAIR) which captures inaccuracy in both easy (endpoint) and difficult (slope) regimes of the stimulus space.

The current experimental design differs from several previous timing studies in at least two respects. First, studies using behaviors such as the Peak Interval task (Catania, 1970; Roberts, 1981) involve response timing rather than the perceptual duration estimation used here. The two may be subserved by different brain structures. Second, to obtain stable psychometric curves, our subjects underwent extensive training. Brain structures involved after such extensive training may differ from those involved early in training (Ponnusamy et al., 2007). In addition to differences with previous tasks, lesion studies in general suffer from at least two drawbacks. First is the possibility of performance recovery owing to potential post-lesion brain reorganization (Yamasaki and Wurtz, 1991). To minimize such effects, we tested subjects only in a short window of time post-lesion. Second is the possibility that a behavior may be subserved by multiple redundant pathways, in which case multiple simultaneous lesions may be required in order to reveal behavioral deficits. Here we lesioned single brain regions per subject.

In our hands, Weber ratios for pure tone frequency discrimination were an order of magnitude higher than those reported in "go/no-go" tasks in which rats report changes in ongoing stimuli (Talwar and Gerstein, 1998; Sloan et al., 2009), perhaps reflecting lower memory and waiting requirements of the latter. In contrast, we found Weber ratios for rats performing duration discrimination to be similar to those reported elsewhere for 


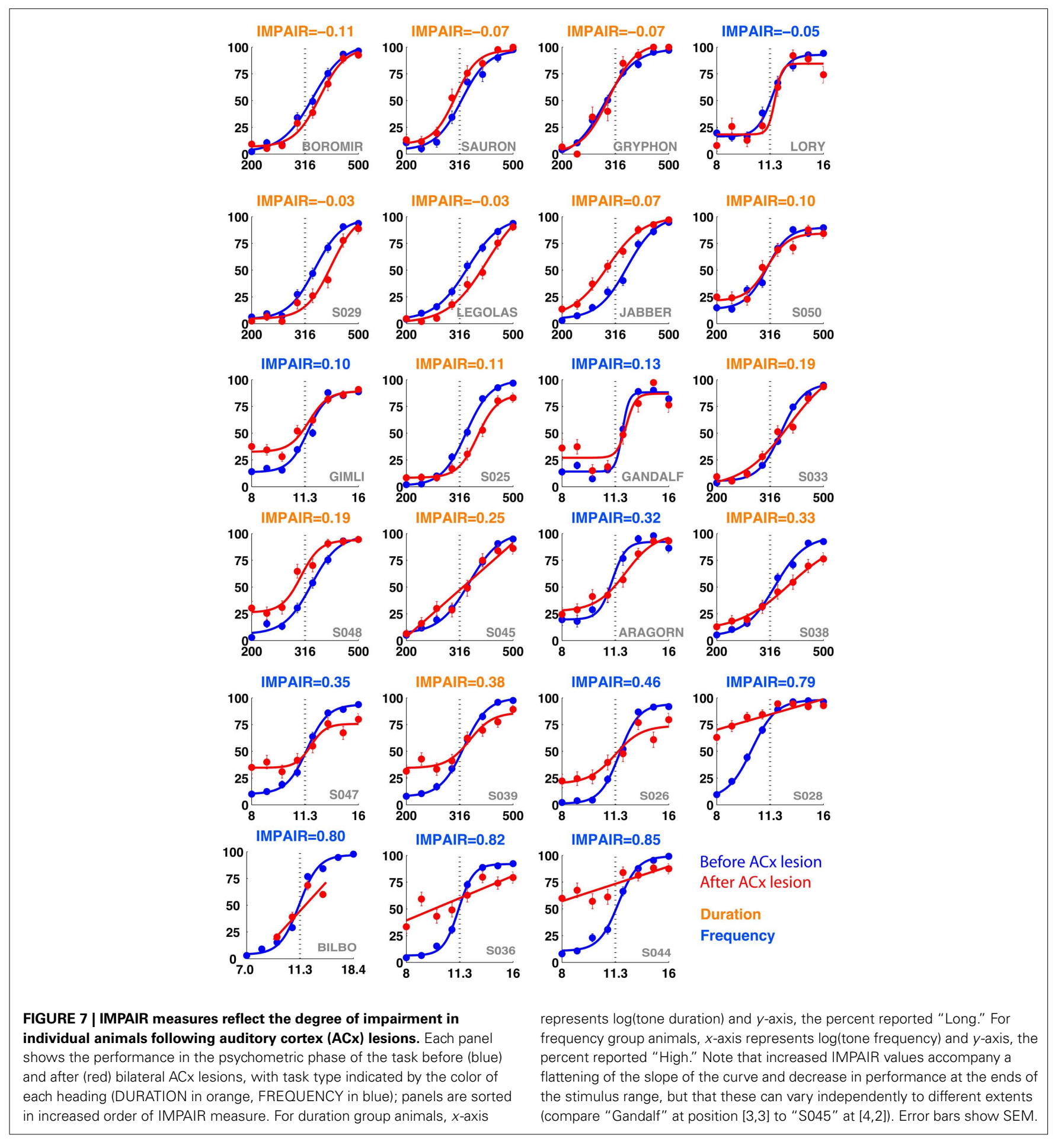

bisection tasks (Church and Deluty, 1977; Orduña et al., 2007, Weber $\approx 0.23$ ).

\section{CONTRIBUTION TO THE KNOWN FUNCTION OF THE RAT AUDITORY CORTEX}

We found that extensive lesions of $\mathrm{ACx}$ produced a much greater performance deficit in subjects discriminating pure tone frequencies than in those discriminating pure tone durations.

Differences in behavioral task design are often offered as an explanation to resolve discrepant results in the literature. Here, we obtained different effects across the two tasks even though the basic task design and all task-related parameters, other than the stimulus feature to be discriminated, were kept equal across the two tasks. To our knowledge, ACx lesions have not previously been performed in duration discrimination tasks. 

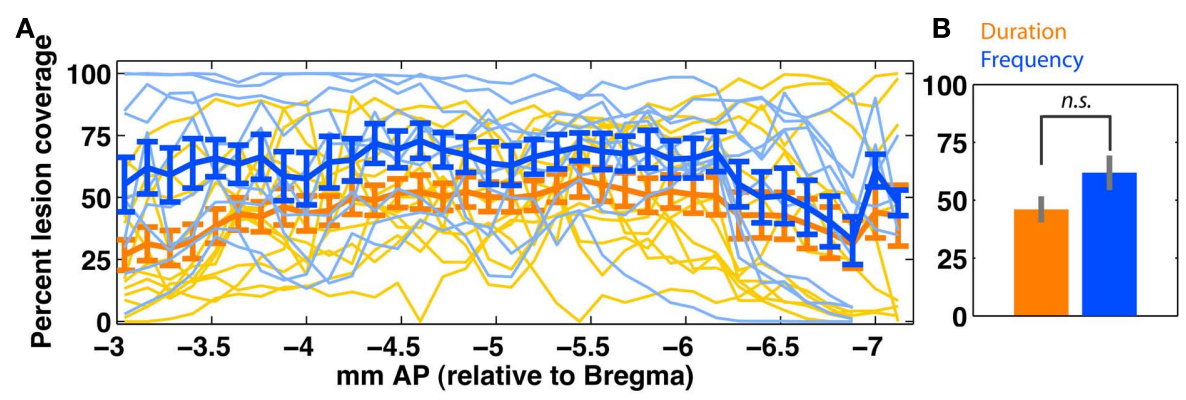

FIGURE 8 | Quantification of bilateral auditory cortex (ACx) lesion extent in duration (orange; $n=13$ ) and frequency (blue; $\boldsymbol{n}=\mathbf{1 0}$ ) groups. (A) Shows slice-wise bilateral coverage. Individual data is shown in thin lines; the group average $( \pm S E M)$ is shown in thick lines. (B) Shows the overall tissue coverage in both groups (mean \pm SEM). A permutation test $(r e p=1000)$ shows that coverage in the two groups is not significantly different $(p>0.1)$. Due to variable tissue coverage, we regressed behavioral impairment against degree of $A C x$ coverage (Figure 9). ACx is defined as areas $\{A 1, A u V$, AuD, TeA\}.

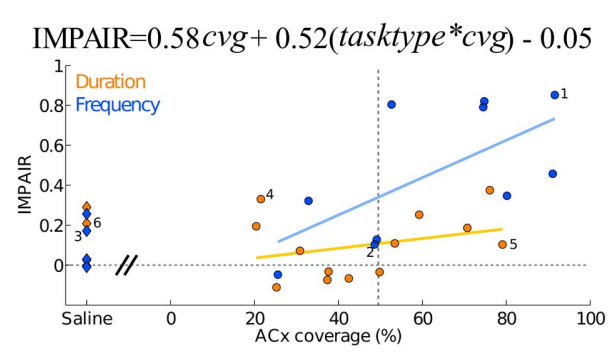

FIGURE 9 |Auditory cortex lesions: impairment relative to the coverage extent of auditory cortex (areas A1, AuD, AuV, and TeA). Each point depicts one animal, the ibotenic acid-injected $(n=23)$ shown as circles and the saline-injected $(n=8)$ as diamonds. A linear regression showed significant effect of an interaction between coverage extent and task type (tasktype ${ }^{*} \mathrm{cvg}=0.52 ; 95 \% \mathrm{Cl}=[0.18,0.85]$ ) in addition to a significant effect of coverage extent ( $c v g=0.58 ; 95 \% \mathrm{Cl}=[0.14,1.02]$ ). Individuals with assigned numbers are shown in detail in Figure 6. Task type was coded as an indicator variable (frequency $=+0.5$, duration $=-0.5$ ).

Our findings with respect to frequency discrimination are consistent with other findings in the literature (Talwar et al., 2001). At this point, we cannot determine whether the deficits in the frequency discriminating group are due to impairments in mnemonic abilities (e.g., memory of task-relevant frequencies) or in non-mnemonic abilities (e.g., ability to compare two frequencies and decide which is higher). Learning increases the number of ACx neurons responding to a task-relevant frequency, with cortex itself likely being the site of plasticity (Buonomano and Merzenich, 1998; Rutkowski and Weinberger, 2005; Froemke et al., 2007). These findings suggest that ACx may be the site of memory of the fiducial frequencies in this task. If this were true, electrophysiological assays of tonotopic representation, performed throughout the training history, could in principle distinguish between neurobiological strategies used to solve the task (e.g., an expansion of representation near the categorization boundary or one near each of the two endpoint stimuli; Han et al., 2007; Kim and Bao, 2008).

While ACx is established as being required for frequency processing, it is presently unclear whether or not it is similarly involved in temporal processing (e.g., storing the memory of duration of

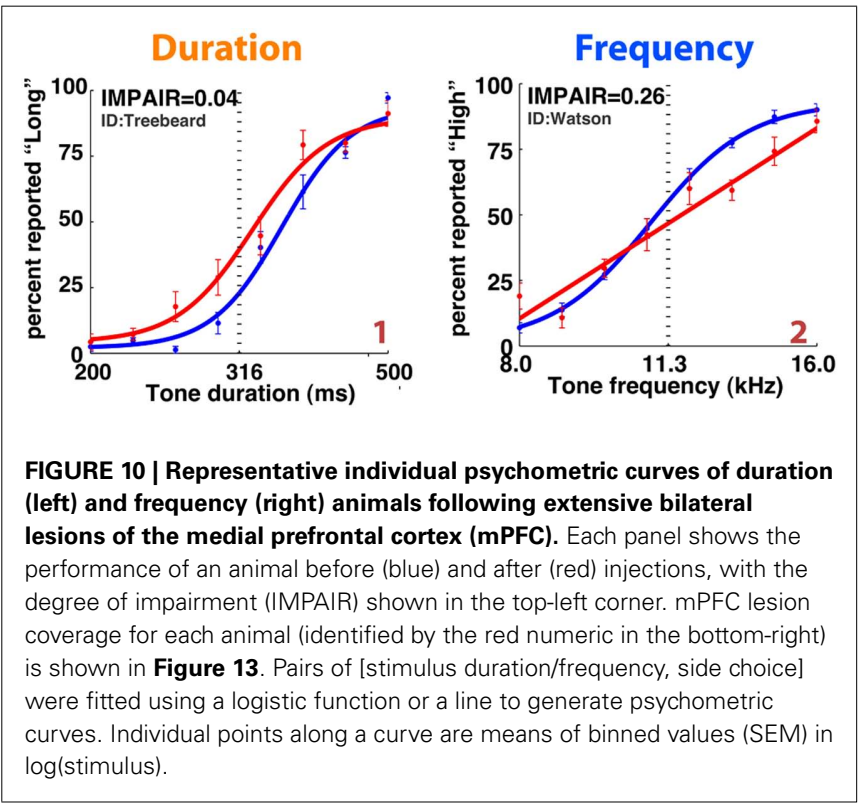

the categorization boundary, as opposed to simply causing the tone to be heard). Neurons in the primate $\mathrm{ACx}$ are responsive to the temporal rate of the stimulus (Beitel et al., 2003), and perceptual learning in an auditory task can lead to improved temporal fidelity in the response of ACx neurons to auditory stimuli (Bao et al., 2004). In our experiments, animals performing the duration discrimination task show significantly less impairment than those discriminating frequency, suggesting that ACX is probably not involved in this capacity. One possibility suggested by these results is that the timing and plasticity required for duration discrimination occurs through a subcortical route, either via the thalamus or midbrain (Figure 14). The former could involve the striatum, a structure implicated in mediating temporal processing (Rao et al., 2001; Meck, 2006). Another possibility is that higher cortical structures, perhaps ones receiving minor projections from the auditory brainstem or striatum (e.g., parietal areas, Leon and Shadlen, 2003; Janssen and Shadlen, 2005), are involved in duration perception. 


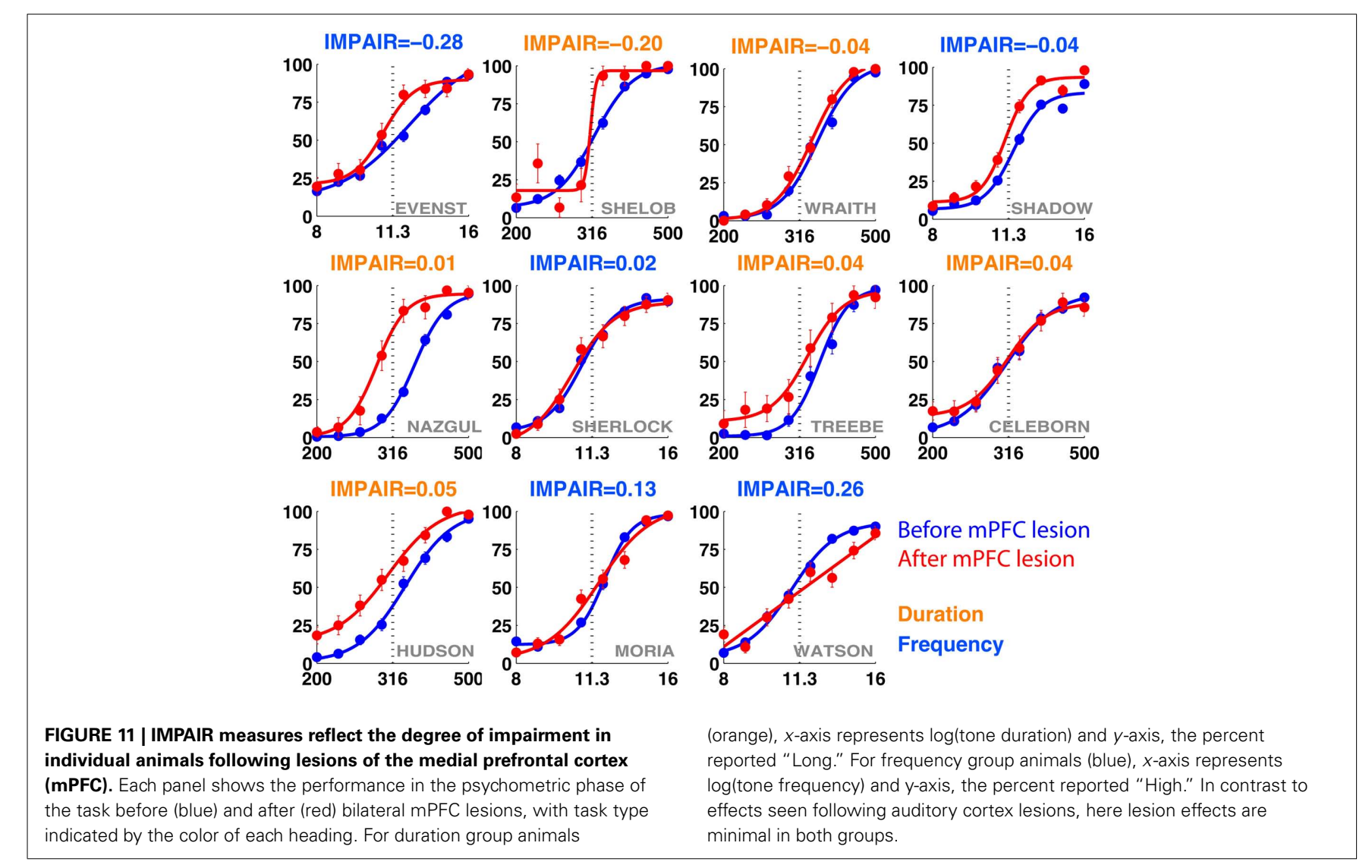

FIGURE 11 | IMPAIR measures reflect the degree of impairment in individual animals following lesions of the medial prefrontal cortex the task before (blue) and after (red) bilateral mPFC lesions, with task type dicated by the color of each heading. For duration group animals
Before MPFC lesion After mPFC lesion

\section{Duration}

Frequency

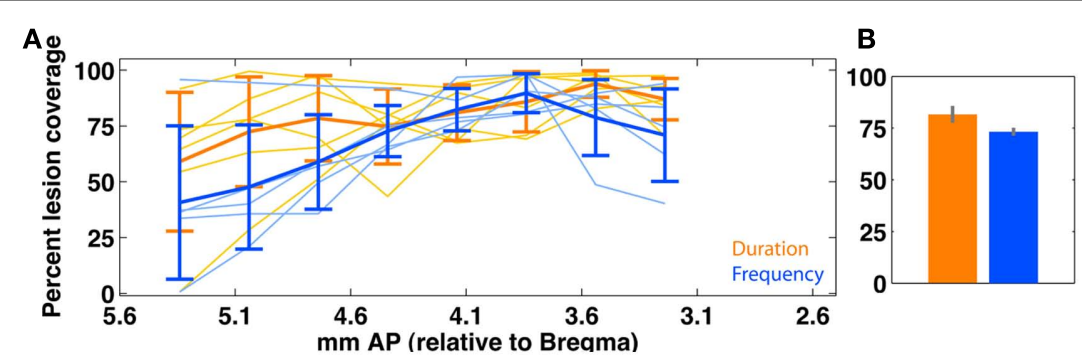

FIGURE 12 | Quantification of bilateral medial prefrontal cortex ( $\mathrm{mPFC}$ ) lesion extent in duration (orange; $n=6$ ) and frequency (blue; $\boldsymbol{n = 5}$ ) groups. (A) Shows slice-wise bilateral coverage. Individual data is shown in thin lines; the group average ( \pm SEM) is shown in thick lines. (B) shows the overall tissue coverage in both groups (mean \pm SEM). A permutation test $(r e p=1000)$ shows that coverage in the two groups is not significantly different $(p>0.1)$. Due to variable tissue coverage, we regressed behavioral impairment against degree of mPFC coverage (Figure 13). mPFC is defined as areas $\{\operatorname{PrL}, I L\}$.
A limitation of our experiment is the incomplete coverage of $\mathrm{ACx}$ in the timing group in general, with the highest coverage being at around $80 \%$ strictly based on brain atlas coordinates. This gap, mainly between -2.5 and $-3.5 \mathrm{~mm}$ AP in the left hemisphere (which in the atlas is marked as areas "S1" and "AuD"), leaves open the possibility that that remaining tissue may be mediating the ability to time sounds. It is also possible that timing may be mediated by higher auditory cortices not as yet identified in the current rat atlas (Kalatsky et al., 2005), that were spared by the toxin. Nevertheless, it is notable that where frequency and timing rats have similar gross coverage (Figure 9), they show very different impairment values. We suggest that in the search for timing-specific brain loci, and in the context of a global picture of audiomotor connectivity (Figure 14), the "corticocortical branch" is likely not the one being used, although other possibilities still exist (see Perspectives).

Several saline-injected animals had impairment values comparable to those injected with ibotenic acid. In contrast, ibotenic acid injections into the $\mathrm{mPFC}$ (next section) resulted in even lower impairment than saline injections in the ACx did. This disparity in impairment suggests that the targeting technique used for ACx lesions may have caused sustained tissue damage unobservable 
by Nissl stain, which resulted in variable impairment. Nevertheless, the impairment observed in the frequency group exceeds that observed in the saline-injected animals, indicating that all impairment seen in these animals is not an artifact of technical limitations. No gliosis was observed at the injection site in any of the saline-injected animals (data not shown); the only tissue damage appeared to be that at the site of the craniotomy.

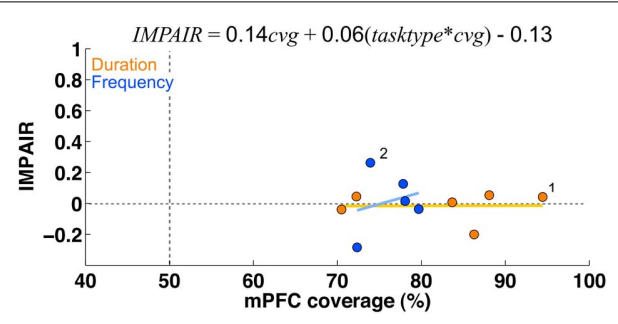

FIGURE 13 | Medial prefrontal cortex lesions: there was little to no impairment in either group following lesions of areas PrL and IL, despite large coverage of these areas. There was also no correlation between impairment and lesion coverage. The graph shows impairment (measured by IMPAIR, $y$-axis) relative to the coverage extent of medial prefrontal cortex ( $x$-axis; defined as areas $\operatorname{PrL}$ and IL). Each point shows data for one ibotenic acid-injected animal ( $n=11$; timing $=6$, frequency $=5$ ). A linear regression showed that neither coverage extent on its own nor in an interaction with task type significantly explained impairment (tasktype* $\mathrm{cvg}$ coefficient $=0.06 ; 95 \% \mathrm{Cl}=[-0.27,0.38] ; \mathrm{cvg}=0.17 ; 95 \% \mathrm{Cl}=[-1.61$, 1.95]); task type was coded as an indicator variable (frequency $=+0.5$, duration $=-0.5$. . No saline controls were conducted for this experiment.

\section{MEDIAL PREFRONTAL CORTEX LESIONS}

In the duration discrimination task, the duration of the pure tone stimulus could in principle be estimated by having the onset of the stimulus trigger the temporal integration of a constant input. This would produce a signal that linearly ramped up over time, therefore acting as a timer. Such temporally ramping signals are commonly seen in the prefrontal cortex of primates (Rainer and Miller, 2002; Brody et al., 2003a,b; Machens et al., 2010). Similarly to primate prefrontal cortex, the rat $\mathrm{mPFC}$ has been proposed to mediate short-term memory (Kolb et al., 1994; Jackson et al., 1998; Ragozzino et al., 2002; Kesner and Ragozzino, 2003; Dalley et al., 2004; Di Pietro et al., 2004; Yoon et al., 2008). Therefore we hypothesized that, following mPFC lesions, we would observe a deficit specifically in the timing group, and not in the frequency group. Our results provide no support for this hypothesis: large lesions of the mPFC had surprisingly little enduring effect in either timing or frequency group. We failed to observe an impairment even within the first 2 days following a lesion; in contrast, performance in the first 3 days following lesions of the ACx still showed a clear impairment in frequency discrimination. Preliminary results from subsequent experiments using muscimol to reversibly inactivate mPFC also failed to show an impairment in duration discrimination (data not shown). The lack of an mPFC requirement for duration discrimination is consistent with our suggestion that the task may be subserved by a subcortical auditory pathway, but do not preclude the possible requirement of other prefrontal areas (e.g., more dorsal aspects).

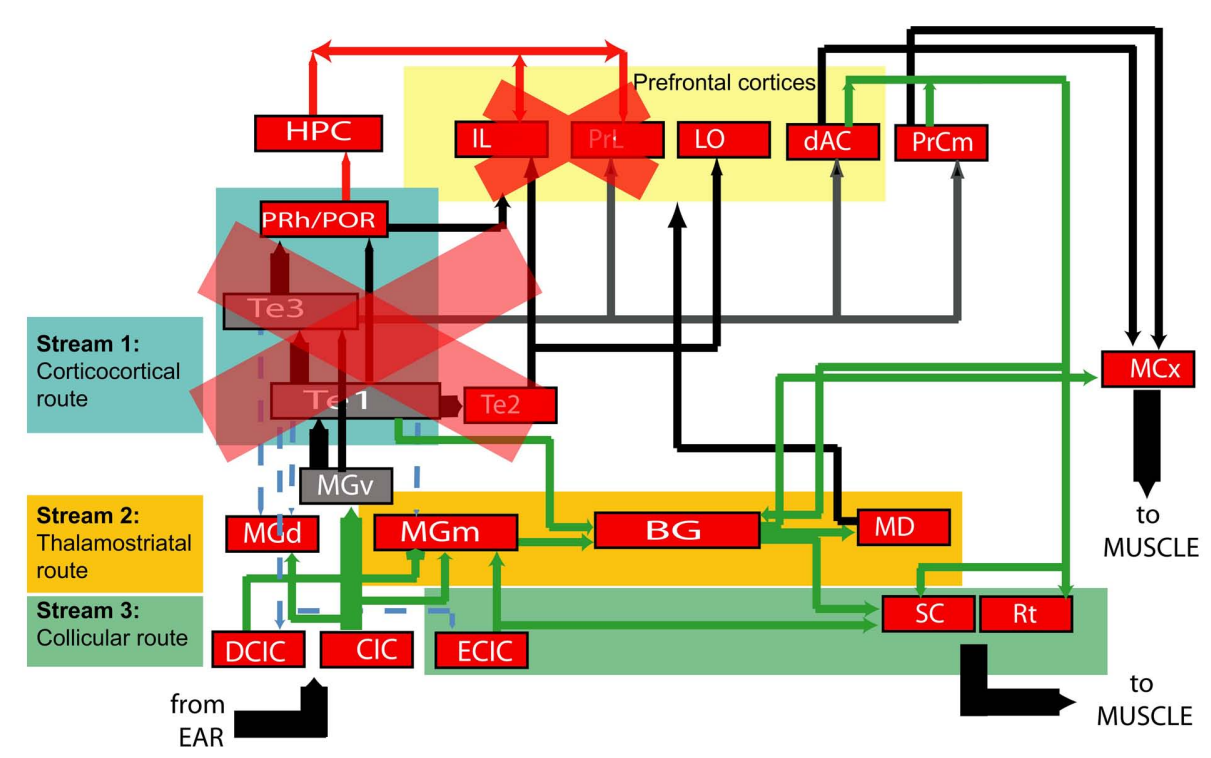

FIGURE 14 | Simplified diagram of neural connections from auditory periphery to the motor system. Each node represents a neural structure of interest to this study, and arrows represent efferent connections. Broadly, there are three possible audiomotor "streams" by which timing and frequency processing could be mediated: the canonical route through sensory cortex (Stream 1 in teal), a thalamostriatal route (Stream 2 in saffron) and a route from the inferior to superior colliculus (Stream 3 in green). Our experiments suggest that the extended auditory cortex is not required for timing in these tasks, and neither is the medial prefrontal cortex. One possibility suggested by these eliminations is that duration perception is mediated by the subcortical streams. An alternate possibility is that cortical structures not targeted by our experiments, but which receive direct input from auditory brainstem, mediate the ability to perceive pure tone durations. Projection key: blue-dashed: corticofugal; red: to/from the hippocampus; green: subcortical; thick black: corticocortical.(Neuroanatomy references: Donoghue and Parham, 1983; Roger and Arnault, 1989; Sesack et al., 1989; Arnault and Roger, 1990; Romanski and LeDoux, 1993; Condé et al., 1995; Burwell and Amaral, 1998; Winer and Schreiner, 2005; García Del Caño et al., 2006). 


\section{PERSPECTIVES}

This work provides the behavioral foundation for a controlled inquiry into the neural mechanisms mediating duration estimation. A behavioral setup similar to ours, with a pair of matched sensory-based tasks, has been previously used as the framework for a brain imaging study of timing in humans (Rao et al., 2001). However, to our knowledge, the paired behavioral approach is the first of its kind to be used for the study of temporal processing in an animal that allows invasive experiments. Interval timing has been studied extensively in the rat (Maricq et al., 1981; Meck, 1996, 2006; Buhusi and Meck, 2005; Drew et al., 2007). The behavioral approach presented here is one where the duration to be estimated is exclusively perceptual, there is a matched control task to account for non-timing aspects of the task, and the auditory nature of the stimulus constrains the search for neural substrates underlying task performance to structures stemming from auditory pathways.

Our results partially prune the components of the neuroanatomical network that specifically mediate the ability to time sound. We show that animals with extensive bilateral damage to the ACx still perform the duration task. Taken together with the lack of impairment following mPFC lesions and the neuroanatomy of audiomotor pathways, the findings suggest that the neural pathway for processing stimulus duration is subcortical. The computation of elapsed duration itself may either occur in subcortical auditory structures, such as the thalamus or inferior colliculus, or in a non-auditory structure, such as the cerebellum (Lewis and Miall, 2003). Another possibility is that timing could involve cortical structures receiving less prominent auditory input - connections omitted from the neuroanatomical diagram (Figure 14) - and which were not the target of our lesions. These structures could process purely sensory information, but may also involve more multimodal cortical structures.

\section{ACKNOWLEDGMENTS}

We thank the technicians for managing the rat training: Joshua Cohen, Glyn Brown, Klaus Osorio. Sebastian Awwad was our system administrator at Princeton University. This work was funded by the Watson School of Biological Sciences, by the Cold Spring Harbor Laboratory Centre for Neural Mechanisms of Cognition, and by the Howard Hughes Medical Institute.

\section{REFERENCES}

Arnault, P., and Roger, M. (1990). Ventral temporal cortex in the rat: connections of secondary auditory areas te2 and te3. J. Comp. Neurol. 302, 110-123.

Bao, S., Chang, E. F., Woods, J., and Merzenich, M. M. (2004). Temporal plasticity in the primary auditory cortex induced by operant perceptual learning. Nat. Neurosci. 7, 974-981.

Beitel, R. E., Schreiner, C. E., Cheung, S. W., Wang, X., and Merzenich, M. M. (2003). Reward-dependent plasticity in the primary auditory cortex of adult monkeys trained to discriminate temporally modulated signals. Proc. Natl. Acad. Sci. U.S.A. 100, 11070-11075.

Boisvert, M. J., and Sherry, D. F. (2006). Interval timing by an invertebrate, the bumble bee bombus impatiens. Curr. Biol. 16, 1636-1640.

Brody, C. D., Hernandez, A., Zainos, A., and Romo, R. (2003a). Timing and neural encoding of somatosensory parametric working memory in macaque prefrontal cortex. Cereb. Cortex 13, 1196-1207.

Brody, C. D., Romo, R., and Kepecs, A. (2003b). Basic mechanisms for graded persistent activity: discrete attractors, continuous attractors, and dynamic representations. Curr. Opin. Neurobiol. 13, 204-211.

Buhusi, C. V., and Meck, W. H. (2005). What makes us tick? Functional and neural mechanisms of interval timing. Nat. Rev. Neurosci. 6, 755-765.

Buonomano, D. V., and Merzenich, M. M. (1998). Cortical plasticity: from synapses to maps. Annu. Rev. Neurosci. 21, 149-186.

Burwell, R. D., and Amaral, D. G. (1998). Cortical afferents of the perirhinal, postrhinal, and entorhinal cortices of the rat. J. Comp. Neurol. 398, 179-205.

Carr, C. E. (1993). Processing of temporal information in the brain. Annu. Rev. Neurosci. 16, 223-243.

Catania, A. C. (1970). The Theory of Reinforcement Schedules, Chapter Reinforcement Schedules and Psychophysical Judgements. New York: Appleton-Century-Crofts, 1-42.

Church, R. M., and Deluty, M.Z. (1977). Bisection of temporal intervals. J. Exp. Psychol. Anim. Behav. Process. 3, 216-228.

Condé, F., Maire-Lepoivre, E., Audinat, E., and Crépel, F. (1995). Afferent connections of the medial frontal cortex of the rat. ii. Cortical and subcortical afferents. J. Comp. Neurol. 352, 567-593.

Dalley, J. W., Cardinal, R. N., and Robbins, T. W. (2004). Prefrontal executive and cognitive functions in rodents: neural and neurochemical substrates. Neurosci. Biobehav. Rev. 28, 771-784.

Di Pietro, N. C., Black, Y. D., GreenJordan, K., Eichenbaum, H. B., and Kantak, K. M. (2004). Complementary tasks to measure working memory in distinct prefrontal cortex subregions in rats. Behav. Neurosci. 118, 1042-1051.

Donoghue, J. P., and Parham, C. (1983). Afferent connections of the lateral agranular field of the rat motor cortex. J. Comp. Neurol. 217, 390-404.
Drew, M. R., Simpson, E. H., Kellendonk, C., Herzberg, W. G., Lipatova, O., Fairhurst, S., Kandel, E. R., Malapani, C., and Balsam, P. D. (2007). Transient overexpression of striatal $\mathrm{d} 2$ receptors impairs operant motivation and interval timing. J. Neurosci. 27, 7731-7739.

Froemke, R. C., Merzenich, M. M., and Schreiner, C. E. (2007). A synaptic memory trace for cortical receptive field plasticity. Nature 450, 425-429.

García Del Caño, G., Gerrikagoitia, I. Alonso-Cabria, A., and MartínezMillán, L. (2006). Organization and origin of the connection from the inferior to the superior colliculi in the rat. J. Comp. Neurol. 499, 716-731.

Gibbon, J. (1977). Scalar expectancytheory and weber's law in animal timing. Psychol. Rev. 84, 279-325.

Han, Y. K., Kover, H., Insanally, M. N., Semerdjian, J. H., and Bao, S. (2007). Early experience impairs perceptual discrimination. Nat. Neurosci. 10, 1191-1197.

Henderson, J., Hurly, T. A., Bateson, M., and Healy, S. D. (2006). Timing in free-living rufous hummingbirds, selasphorus rufus. Curr. Biol. 16, 512-515.

Jackson, P. A., Kesner, R. P., and Amann, K. (1998). Memory for duration: role of hippocampus and medial prefrontal cortex. Neurobiol. Learn. Mem. 70, 328-348.

Janssen, P., and Shadlen, M. N. (2005). A representation of the hazard rate of elapsed time in macaque area lip. Nat. Neurosci. 8, 234-241.
Kalatsky, V. A., Polley, D. B., Merzenich, M. M., Schreiner, C. E., and Stryker, M. P. (2005). Fine functional organization of auditory cortex revealed by fourier optical imaging. Proc. Natl. Acad. Sci. U.S.A. 102, 13325-13330.

Kesner, R. P., and Ragozzino, M. E. (2003). The role of the prefrontal cortex in object-place learning: a test of the attribute specificity model. Behav. Brain Res. 146, 159-165.

Kim, H., and Bao, S. (2008). Distributed representation of perceptual categories in the auditory cortex. $J$. Comput. Neurosci. 24, 277-290.

Kolb, B., Buhrmann, K., McDonald, R., and Sutherland, R. J. (1994). Dissociation of the medial prefrontal, posterior parietal, and posterior temporal cortex for spatial navigation and recognition memory in the rat. Cereb. Cortex 4, 664-680.

Leon, M. I., and Shadlen, M. N. (2003). Representation of time by neurons in the posterior parietal cortex of the macaque. Neuron 38, 317-327.

Lewis, P. A., and Miall, R. C. (2003). Distinct systems for automatic and cognitively controlled time measurement: evidence from neuroimaging. Curr. Opin. Neurobiol. 13, 250-255.

Machens, C. K., Romo, R., and Brody, C. D. (2010). Functional, but not anatomical, separation of "what" and "when" in prefrontal cortex. $J$. Neurosci. 30, 350-360.

Maricq, A. V., Roberts, S., and Church, R. M. (1981). Methamphetamine and time estimation. J. Exp. Psychol. Anim. Behav. Process. 7, 18-30. 
Mauk, M. D., and Buonomano, D. V. (2004). The neural basis of temporal processing. Annu. Rev. Neurosci. 27, 307-340.

Meck, W. H. (1986). Affinity for the dopamine $\mathrm{d} 2$ receptor predicts neuroleptic potency in decreasing the speed of an internal clock. Pharmacol. Biochem. Behav. 25, 1185-1189.

Meck, W. H. (1996). Neuropharmacology of timing and time perception. Brain Res. Cogn. Brain Res. 3, 227-242.

Meck, W. H. (2006). Neuroanatomical localization of an internal clock: a functional link between mesolimbic, nigrostriatal, and mesocortical dopaminergic systems. Brain Res. 1109, 93-107.

Nenadic, I., Gaser, C., Volz, H.-P. P., Rammsayer, T., Häger, F., and Sauer, H. (2003). Processing of temporal information and the basal ganglia: new evidence from fmri. Exp. Brain Res. 148, 238-246.

Orduña, V., Hong, E., and Bouzas, A. (2007). Interval bisection in spontaneously hypertensive rats. Behav. Processes 74, 107-111.

Paxinos, G., and Watson, C. (2007). The Rat Brain in Stereotaxic Coordinates. London: Academic Press.

Ponnusamy, R., Poulos, A. M., and Fanselow, M. S. (2007). Amygdala-dependent and amygdala-independent pathways for contextual fear conditioning. Neuroscience 147, 919-927.
Press, W. H., Teukolsky, S. A., Vetterling, W. T., and Flannery, B. P. (1992). Numerical Recipes in C: The Art of Scientific Computing, Chapt. 15. Cambridge: Cambridge University Press, 656-706.

Ragozzino, M. E., Detrick, S., and Kesner, R. P. (2002). The effects of prelimbic and infralimbic lesions on working memory for visual objects in rats. Neurobiol. Learn. Mem. 77, 29-43.

Rainer, G., and Miller, E. K. (2002). Timecourse of object-related neural activity in the primate prefrontal cortex during a short-term memory task. Eur. J. Neurosci. 15, 1244-1254.

Rao, S. M., Mayer, A. R., and Harrington, D. L. (2001). The evolution of brain activation during temporal processing. Nat. Neurosci. 4, 317-323.

Roberts, S. (1981). Isolation of an internal clock. J. Exp. Psychol. Anim. Behav. Process. 7, 242-268.

Roger, M., and Arnault, P. (1989). Anatomical study of the connections of the primary auditory area in the rat. J. Comp. Neurol. 287, 339-356.

Romanski, L. M., and LeDoux, J. E. (1993). Information cascade from primary auditory cortex to the amygdala: corticocortical and corticoamygdaloid projections of temporal cortex in the rat. Cereb. Cortex 3, 515-532.

Rutkowski, R. G., and Weinberger, N. M. (2005). Encoding of learned importance of sound by magnitude of representational area in primary auditory cortex. Proc. Natl. Acad. Sci. U.S.A. 102, 13664-13669.

Sesack, S. R., Deutch, A. Y., Roth, R. H., and Bunney, B. S. (1989). Topographical organization of the efferent projections of the medial prefrontal cortex in the rat: an anterograde tract-tracing study with phaseolus vulgaris leucoagglutinin. J. Comp. Neurol. 290, 213-242.

Sloan, A. M., Dodd, O. T., and Rennaker, R. L. II. (2009). Frequency discrimination in rats measured with tonestep stimuli and discrete pure tones. Hear. Res. 251, 60-69.

Talwar, S. K., and Gerstein, G. L. (1998) Auditory frequency discrimination in the white rat. Hear. Res. 126, 135-150.

Talwar, S. K., Musial, P. G., and Gerstein, G. L. (2001). Role of mammalian auditory cortex in the perception of elementary sound properties. $J$. Neurophysiol. 85, 2350-2358.

Wager-Smith, K., and Kay, S. A. (2000). Circadian rhythm genetics: from flies to mice to humans. Nat. Genet. 26, 23-27.

Ward, R. D., Kellendonk, C., Simpson, E. H., Lipatova, O., Drew, M. R., Fairhurst, S., Kandel, E. R., and Balsam, P. D. (2009). Impaired timing precision produced by striatal $\mathrm{d} 2$ receptor overexpression is mediated by cognitive and motivational deficits. Behav. Neurosci. 123, 720-730.
Winer, J. A., and Schreiner, C. (2005). The Inferior Colliculus: With 168 Illustrations. New York, NY: Springer.

Yamasaki, D. S., and Wurtz, R. H. (1991). Recovery of function after lesions in the superior temporal sulcus in the monkey. J. Neurophysiol. $66,651-673$.

Yoon, T., Okada, J., Jung, M. W., and Kim, J. J. (2008). Prefrontal cortex and hippocampus subserve different components of working memory in rats. Learn. Mem. 15, 97-105.

Conflict of Interest Statement: The authors declare that the research was conducted in the absence of any commercial or financial relationships that could be construed as a potential conflict of interest.

Received: 03 June 2011; accepted: 13 August 2011; published online: 27 September 2011.

Citation: Pai S, Erlich JC, Kopec C and Brody CD (2011) Minimal impairment in a rat model of duration discrimination following excitotoxic lesions of primary auditory and prefrontal cortices. Front. Syst. Neurosci. 5:74. doi: 10.3389/fnsys.2011.00074

Copyright (C) 2011 Pai, Erlich, Kopec and Brody. This is an open-access article subject to a non-exclusive license between the authors and Frontiers Media SA, which permits use, distribution and reproduction in other forums, provided the original authors and source are credited and other Frontiers conditions are complied with. 